\title{
An Algorithm Based on Combining hs-cTnT and H-FABP for Ruling Out Acute Myocardial Infarction
}

\author{
César Navarro ${ }^{1}$, Mary Jo Kurth ${ }^{2}$, Mark Ruddock ${ }^{2}$, Sam Fishlock ${ }^{1}$ and James McLaughlin ${ }^{1}$ \\ ${ }^{1}$ Connected Health Innovation Centre (CHIC) NIBEC, Ulster University, Newtownabbey, UK \\ ${ }^{2}$ Randox Laboratories Ltd, Crumlin, UK
}

\begin{abstract}
Our previous work demonstrated that algorithms combining high sensitivity cardiac troponin $T(h s-c T n T)$ and heart-type fatty acid-binding protein (H-FABP) may help in ruling out Acute Myocardial Infarction (AMI). For those algorithms, the hs-cTnT thresholds were adopted from the ESC guidelines. This time, we present a data-driven approach that also explores hs-cTnT thresholds.

The results show a significant improvement when compared to previous algorithms reported. Using a cohort of $n=360$ patients (288 Non-AMI and 72 AMI), a ruleout algorithm used at presentation identified more low-risk patients who presented with chest pain of suspected cardiac origin than the standard ESC algorithm: (199/288 (69.1\%) vs. 83/288 (28.8\%) ( $p<0.0005))$, respectively.

According to our data, our algorithm at the emergency department, would identify additional non-AMI patients in comparison to the ESC algorithm, potentially reducing the number of hospital admissions by $42 \%$.
\end{abstract}

\section{Introduction}

Chest pain is a common complaint for presentation at the emergency department and over 6 million patients present annually in the United States [1]. For patients that are admitted, only $10 \%$ to $13 \%$ will have a confirmed AMI [2]. In England and Wales, 700,000 patients annually present with chest pain to the emergency department, and two thirds of these patients are admitted [3]. In Europe, the situation is no better [4]. Unfortunately, many of the diagnostic tests that are currently available do not have either the sensitivity and specificity to effectively rule-out MI at presentation [5]. There are considerable logistical and financial burdens linked to the management of ECG negative patients who often have to wait for a second and third cTnT test. Furthermore, uncertainty with the discharge of these patients can be associated with a 5-fold increase in their mortality and morbidity [6].

Our group developed algorithms based on decision trees combining values of high sensitivity troponin $\mathrm{T}$ (hs-cTnT) and heart-type fatty acid-binding protein (H-FABP) for a safe and early release of patients with suspected AMI. HFABP was selected as an optimal biomarker to help hscTnT over other biomarkers [7]. The analysis demonstrated that hs-cTnT consistently had a higher Area Under the Receiver Operating characteristic Curve (AUROC) than the other biomarkers but was not statistically different to H-FABP at presentation [8] and at 3 and 6 hours [7]. $\mathrm{H}-\mathrm{FABP}$ was also a significant predictor of AMI according to logistic regression models [7]. However, H-FABP when acting as a sole biomarker did not outperform hs-cTnT. It is important to keep in mind the following question: can an alternative biomarker outperform hs-cTnT when hs-cTnT is used to identify a case as AMI?

Further, we explored the question: in which cases could H-FABP help in the diagnosis of AMI when combined with hs-cTnT? Body et al. [9] have proposed diagnostic models for ruling out AMI. We have been developing rule-out algorithms especially in cases when there is a high baseline hs-cTnT and taking into account that $\mathrm{H}-$ FABP generally peaks earlier than hs-cTnT in AMI [7].

The previous algorithms show thresholds and rules for H-FABP leaving the hs-cTnT thresholds unaltered to those recommended by the European Society of Cardiology (ESC) [8]. This study aimed to explore further hs-cTnT thresholds in combination with H-FABP. Also, the presence of ST shifts is considered.

\section{Methods}

\subsection{Recruitment of patients and Data}

Consecutive patients (age $>18$ years) with chest pain of presumed ischaemic origin were recruited at Craigavon Area and Daisy Hill hospitals in Northern Ireland between October 2009 and October 2011 [8]. More details about the ethical approval, demographics, biomarkers measured at different time points including hs-cTnT and H-FABP have been previously reported [8].

For the AMI cases, only non-ST Elevation MI 
(NSTEMI) were retrospectively considered since ST Elevation MI (STEMI) cases are treated by PCI (where it is available) and would not routinely have biomarkers measured.

Data from 360 cases for presentation (288 Non-AMI and 72 AMI) and 320 cases at 1 hour (254 Non-AMI and 66 AMI) from admission were retrospectively studied. There were no missing values for the considered biomarkers.

\subsection{The statistical analysis}

The statistical analyses used for comparing algorithms was the Net Reclassification Improvement (NRI) [10].

\subsection{ESC Algorithms}

The ESC rule-out algorithms [11] for presentation and for 1 hour (that use hs-cTnT) were used to compare against the proposed algorithms.

ESC guidelines for suspected NSTEMI suggest rule-out for (i) hs-cTnT $<5 \mathrm{ng} / \mathrm{l}$ at presentation (t=0 h); (ii) or hs$\mathrm{cTnT}<12 \mathrm{ng} / \mathrm{l}$ at presentation with a delta change $<3 \mathrm{ng} / \mathrm{l}$ at $\mathrm{t}=1 \mathrm{~h}$. For suspected NSTEMI rule-in (i) hs-cTnT $>52$ $\mathrm{ng} / \mathrm{l}$ at $\mathrm{t}=0 \mathrm{~h}$, (ii) or hs-cTnT delta change $(\mathrm{t}=1 \mathrm{~h})>5 \mathrm{ng} / \mathrm{l}$.

\subsection{Exploratory analyses}

hs-cTnT and H-FABP were selected from other biomarkers for ruling-out AMI from the data provided [7]. Thus, the building of the algorithms aimed for sensitivities as close as possible to 1.000 and high specificity.

A number of algorithms based on decision trees were further explored [12]. Raised values of biomarkers were generally in keeping with AMI. However, that was not always the case in the dataset. There were also cases of low values for the biomarkers which were diagnosed AMI.

\section{Results}

\subsection{Proposed 'rule-out" at presentation}

Two thresholds for hs-cTnT (9.5 and $12.36 \mathrm{ng} / \mathrm{l})$ at presentation were used in conjunction with a cut-off for $\mathrm{H}$ FABP of $2.88 \mu \mathrm{g} / \mathrm{l}$ to separate two potential situations when AMI was suspected:

(a) If $\mathrm{H}-\mathrm{FABP} \geq 2.88 \mu \mathrm{g} / \mathrm{l}$ and If hs-cTnT $<9.5 \mathrm{ng} / \mathrm{l}$ then rule-out AMI.

(b) If H-FABP $<2.88 \mu \mathrm{g} / \mathrm{l}$ the higher threshold of 12.36 $\mathrm{ng} / \mathrm{l}$ for hs-cTnT was used. If hs-cTnT is lower, then ruleout AMI. The rationale for these two sub-algorithms at presentation was that an elevated H-FABP value would be in keeping with its earlier increase when hs-cTnT was beginning to rise justifying a lower threshold. Additionally, for this second branch in the algorithm (a higher threshold for
hs-cTnT), the presence/absence of ST shifts was employed to aid in the decision:

(c) if there were no ST shifts then AMI was ruled-out.

Results for these algorithms with and without ST shifts are in table 1 (algorithms 1 and 2, respectively).

\subsection{Proposed 'rule-out" at 1 hour}

For the proposed algorithm at 1 hour there were two measurements included for each biomarker ( 0 and 1 hour). The two biomarker measurements were used to calculate an increase (if any), in absolute values for differences (deltas) and averages. For identifying a possible MI (not rule-out MI) each of the following criteria (a), (b) and (c) had to occur:

(a) any of the H-FABP elevated $(\geq 0.95 \mu \mathrm{g} / \mathrm{l})$ and,

(b) average of hs-cTnT elevated $(>5.5 \mathrm{ng} / \mathrm{l})$ and,

(c) hs-cTnT at 1 hour should be elevated $(>12.52 \mathrm{ng} / \mathrm{l})$ or its increase be $>10 \%$ of the average. However, there were two scenarios where high hs-cTnT or steady hs-cTnT needed to be further investigated when H-FABP values were low. The additional checks for these two particular scenarios to eliminate the suspicion of an AMI (and confirm a rule-out) were:

(d) average of hs-cTnT elevated ( $>50 \mathrm{ng} / \mathrm{l})$ but average of $\mathrm{H}-\mathrm{FABP}<1.7 \mu \mathrm{g} / \mathrm{l}$ or

(e) absolute value of delta hs-cTnT $<2 \%$ of average when average of H-FABP $<1.8 \mu \mathrm{g} / \mathrm{l}$.

Results for this algorithm are in table 1 (algorithm 6).

\subsection{Optimised hs-cTnT-only algorithms}

Algorithms for presentation based on hs-cTnT-only were also optimised to the dataset. Rule-out for hs-cTnT $<9.5 \mathrm{ng} / \mathrm{l}$ at presentation $(\mathrm{t}=0 \mathrm{~h})$. There could be an additional check if ST shifts are considered: their absence confirms the non-AMI suggestion by the biomarker. Results are presented in table 1 (algorithms 3 and 4).

\subsection{Comparisons}

Reference algorithms A and B were previously reported [8] where the thresholds for hs-cTnT were not explored and left as suggested by the ESC guidelines.

The NRI is presented in table 1. For instance, algorithm 5 (ESC at presentation) is more sensitive than algorithm A (net gain with MI of 0.042) but less specific (negative net gain with non-MI of -0.368), and the probability of $|\mathrm{NRI}|$ (absolute value of the sum of the two net gains) greater than $|-0.327|$ is low $(p<0.0005)$ if the hypothesis of NRI zero is assumed. Thus, it can be concluded that these two algorithms are statistically different in terms of NRI. Additionally, a negative NRI shows no overall improvement over the reference algorithm. 
Table 1. Comparisons of the proposed algorithms at presentation $(n=360,72$ AMI) and at 1 hour $(n=320,66$ AMI).

\begin{tabular}{|c|c|c|c|c|c|c|c|c|c|c|c|c|}
\hline Algorithm & $\mathrm{TP}$ & TN & FP & $\mathrm{FN}$ & Sen & Spec & PPV & NPV & $\begin{array}{l}\text { Net gain } \\
\text { AMI }\end{array}$ & $\begin{array}{l}\text { Net gain } \\
\text { non-AMI }\end{array}$ & NRI & $\mathrm{p}$ \\
\hline \multicolumn{13}{|l|}{ Oh (presentation ) } \\
\hline (A) H-FABP \& hs-cTnT* & 69 & 189 & 99 & 3 & 0.958 & 0.656 & 0.411 & 0.984 & NA & NA & NA & NA \\
\hline (1) H-FABP \& hs-cTnT ${ }^{s}$ & 71 & 192 & 96 & 1 & 0.986 & 0.667 & 0.425 & 0.995 & 0.028 & 0.010 & 0.038 & 0.131 \\
\hline (2) H-FABP \& hs-cTnT & 69 & 199 & 89 & 3 & 0.958 & 0.691 & 0.437 & 0.985 & 0 & 0.035 & 0.035 & 0.008 \\
\hline (3) Optimised hs-cTnT ${ }^{s}$ & 71 & 171 & 117 & 1 & 0.986 & 0.594 & 0.378 & 0.994 & 0.028 & -0.063 & -0.035 & 0.232 \\
\hline (4) Optimised hs-cTnT & 69 & 177 & 111 & 3 & 0.958 & 0.613 & 0.383 & 0.983 & 0 & -0.042 & -0.042 & 0.040 \\
\hline (5) ESC rule-out & 72 & 83 & 205 & 0 & 1.000 & 0.288 & 0.260 & 1.000 & 0.042 & -0.368 & -0.327 & 0.000 \\
\hline \multicolumn{13}{|l|}{$0 \mathrm{~h}-1 \mathrm{~h}$} \\
\hline (B) H-FABP \& hs-cTnT* & 66 & 181 & 73 & 0 & 1.000 & 0.713 & 0.475 & 1.000 & NA & NA & NA & NA \\
\hline (6) H-FABP \& hs-cTnT & 66 & 181 & 73 & 0 & 1.000 & 0.713 & 0.475 & 1.000 & 0 & 0 & 0 & 1 \\
\hline (7) ESC rule-out & 63 & 175 & 79 & 3 & 0.955 & 0.689 & 0.444 & 0.983 & -0.045 & -0.026 & -0.069 & 0.031 \\
\hline
\end{tabular}

n: number of patients; AMI: Acute Myocardial Infarction; $\left({ }^{s}\right)$ : ST-shifts; $\left({ }^{*}\right)$ : ESC thresholds for hs-cTnT; TP: true positives; TN: true negatives; FN: false negatives; FP: false positives; Sen: sensitivity; Spec: specificity; PPV: positive predictive value; NPV: negative predictive value; NA: Non-Applicable as it is the reference algorithm; h: hour

\section{Discussion}

Table 1 presents the results. The ESC algorithm for presentation (algorithm 5) [11], based only on hs-cTnT, exhibit the maximum possible sensitivity (detecting all the AMI cases without leaving any false negatives) at the expense of admitting and treating 205 out of 288 non-AMI cases, exhibiting a low specificity of 0.288 . Our previous algorithm (referred to as algorithm A) combining hs-cTnT and H-FABP used thresholds for hs-cTnT from the ESC guidelines [8]. The specificity was increased to 0.656 at the expense of lowering the sensitivity to 0.958 .

The newer algorithms, where the thresholds for hscTnT were also driven by the data analysis, show an improvement for the presentation $(\mathrm{t}=0 \mathrm{~h})$ stage: algorithm 1 demonstrated the highest sensitivity (0.986) and NPV (0.995) with good specificity (0.667). There was one falsenegative: a 53-year-old patient who presented within 1.25 hours from onset of chest pain, the hs-cTnT value was 5.66 ng/l and H-FABP was $0.98 \mu \mathrm{g} / \mathrm{l}$ without ST shifts. Removing the check of ST shifts from the decision tree (algorithm 2) increased the specificity at the expense of 3 false negatives. In both cases the NRI was positive, an improvement, although the algorithm without the ST-shifts checks is the only statistically different from algorithm A.

The ESC algorithm ruled-out AMI in only 83 out of 288 patients in comparison to algorithm 2 which ruled-out 199 patients. Additionally, to the 72 AMI cases, this algorithm would have suggested admitting 205 additional patients when is not required. Thus the total number of admissions would have reached 277. Algorithm 2 would have ruledout 199 out of 288 patients (89 false positives) and admit 69 AMI patients; the total of admissions would have fallen to 158 . However, there were 3 false negatives. Thus, there would have been 116 fewer admissions from a total of 277 which represents $42 \%$ reduction. The NRI for the comparison of these two algorithms is $-0.361(\mathrm{p}<0.0005)-$ not shown in table 1 .

Optimising the decision tree for hs-cTnT-only did not result in a significant change in the sensitivity, but there was a substantial decrease in specificity. False negatives were also detected. The NRI was inconclusive for algorithm 3 when compared to reference algorithm A.

At 1 hour, the proposed algorithms provided no false negatives. Interestingly, when the ESC rule-out was applied to the data, it resulted in 3 false negatives with decreased sensitivity, specificity, PPV and NPV with a negative NRI $(-0.069, \mathrm{p}=0.031)$. Varying the thresholds for hscTnT did not change the results obtained by the reference algorithm B. Also ST shifts did not improve the results.

The current study improves on that of McMahon et al. [13] indicating that H-FABP and cTn in combination can be used to rule out MI, by including hs-cTn and focusing on earlier time points achieving $99.5 \%$ NPV at presentation. Algorithm 1 had comparable sensitivity and NPV to no ECG changes, hs-cTnT and H-FABP [14] but had increased specificity and PPV. The current decision tree combination would have enabled at least two thirds of the patients to be identified as non-AMI at presentation compared to $9.8 \%$ at $2 \mathrm{hr}$ in the ASPECT Study [15], 27.7\% using hs-cTnT at $0 \mathrm{hr}$ using LoD as cut-off (3 ng/l) [16] and $48.8 \%$ using negative ECG, hs-cTnT and H-FABP at $0 \mathrm{hr}$ [14]. The MACS rule, comprising eight variables including hs-cTnT and H-FABP reduced hospital admissions by over $25 \%$ [9]. The current study identified $70 \%$ (181/254) of patients at 1 hour as non-AMI with no false negatives compared to the recent TRAPID-AMI study that also used a 0 and 1-hour algorithm, which ruled out $63.4 \%$ of patients but had 7 false negatives $[17,18]$.

The proposed algorithms could assist in the identifica- 
tion of non-AMI patients. These patients should be investigated for other causes of their chest pain, or indeed discharged ensuring appropriate care and efficient use of resources. Body et al. have suggested savings of over $£ 300$ (€374) per presenting patient with chest pain [19].

\section{Conclusions}

H-FABP has shown promise among other biomarkers investigated to pair with hs-cTnT as a rule-out test for suspected AMI patients. The analyses presented demonstrates that varying thresholds for hs-cTnT to different values as those suggested by the ESC guidelines provides a statistically significant improvement to the results previously reported on the same dataset. Also, the occurrence of ST shift was explored but no conclusive effects were observed.

Although the results are encouraging, a larger multicentre study should be conducted to verify the results. Additionally, false negatives, exhibiting low values of hscTnT and H-FABP, would require further investigation.

\section{Acknowledgments}

INVEST NI, the funders of this research.

\section{References}

[1] Keller T, Zeller T, Peetz D, Tzikas S, Roth A et al. Sensitive troponin I assay in early diagnosis of acute myocardial infarction. N Engl J Med 2009;361:868-877.

[2] Pope J, Aufderheide T, Ruthazer R, Woolard R et al. Missed diagnoses of acute cardiac ischemia in the emergency department. N Engl J Med 2000;34216:1163-1170.

[3] Goodacre S, Cross E, Arnold J, Angelini K, Capewell S, Nicholl J. The health care burden of acute chest pain. Heart 2005;91:229-230.

[4] Bayón Fernández J, Alegría Ezquerra E, Bosch Genover X, Cabadés O'Callaghan A, Iglesias Gárriz I et al. Chest pain units. Organization and protocol for the diagnosis of acute coronary syndromes. Rev Esp Cardiol 2002;55:143-154.

[5] Goldman L, Kirtane A. Triage of patients with acute chest pain and possible cardiac ischemia: The elusive search for diagnostic perfection. Ann Intern Med 2003;139:987-995.

[6] Collinson P, Boa F, Gaze D. Measurement of cardiac troponins. Ann Clin Biochem 2001;423449.

[7] Navarro C, Kurth M, McEneaney DJ, McLaughlin J. A feature selection for detection of non ST elevation myocardial infarction. In Proceedings of Computing in Cardiology. IEEE, 2016; 217-220.

[8] Navarro CO, Kurth M, Lamont JV, Menown IB, Ruddock MW et al. Diagnostic performance of a combination biomarker algorithm for rule-out of acute myocardial infarction at time of presentation to the emergency department, using heart-type fatty acid-binding protein and highsensitivity troponin T tests. Journal of Clinical and Experimental Cardiology 2018;9:1-8.
[9] Body R, Carley S, McDowell G, Pemberton P, Burrows G et al. The Manchester Acute Coronary Syndromes (MACS) decision rule for suspected cardiac chest pain: derivation and external validation. Heart 2014;100:1462-1468.

[10] Pencina M, D'Agostino R Sr, D'Agostino R Jr, Vasan R. Evaluating the added predictive ability of a new marker: From area under the ROC curve to reclassification and beyond. Stat Med 2008;27:157-172.

[11] Roffi M, Patrono C, Collet J, Mueller C, Valgimigli M et al. 2015 ESC guidelines for the management of acute coronary syndromes in patients presenting without persistent ST-segment elevation: Task force for the management of acute coronary syndromes in patients presenting without persistent ST-segment elevation of the European Society of Cardiology (ESC). Eur Heart J 2016;37:267-315.

[12] Zhelev Z, Hyde C, Youngman E, Rogers M, Fleming S et al. Diagnostic accuracy of single baseline measurement of elecsys troponin $\mathrm{T}$ high-sensitive assay for diagnosis of acute myocardial infarction in emergency department: systematic review and meta-analysis. BMJ 2015;350:h15.

[13] McMahon C, Lamont J, Curtin E, McConnell R, Crockard $\mathrm{M}$ et al. Diagnostic accuracy of heart-type fatty acidbinding protein for the early diagnosis of acute myocardial infarction. Am J Emerg Med 2012;30:267-74.

[14] Body R, Carley S, Burrows G, Pemberton P, MackwayJones K. Combining heart fatty acid binding protein and high sensitivity troponin in the emergency department. 14th international conference on emergency medicine. Acad Emerg Med 2012;19:748-749.

[15] Than M, Cullen L, Reid C, Lim S, Aldous S et al. A 2-h diagnostic protocol to assess patients with chest pain symptoms in the asia-pacific region (ASPECT): a prospective observational validation study. Lancet 2011;26:1077-1084.

[16] Body R, Carley S, McDowell G, Jaffe A, France M et al. Rapid exclusion of acute myocardial infarction in patients with undetectable troponin using a high sensitivity assay. J Am Coll Cardiol 2011;58:1332-1339.

[17] Charpentier S, Peschanski N, Chouihed T, Ray P, Chenevier-Gobeaux C. Multicenter evaluation of a 0 hour/1-hour algorithm in the diagnosis of myocardial infarction with high-sensitivity cardiac troponin T. Ann Emerg Med 2016;67:793-794.

[18] Mueller C, Giannitsis E, Christ M, Ordóñez-Llanos J et al. Multicenter evaluation of a 0-hour/1-hour algorithm in the diagnosis of myocardial infarction with high-sensitivity cardiac troponin T. Ann Emerg Med 2016;68:76-87.

[19] Body R, Dixon D, Burrows G, Cook G, Lewis P. Economic evaluation of heart fatty acid binding protein based protocol for rapid chest pain assessment. 14th international conference on emergency medicine. Acad Emerg Med 2012; 19:746-747.

Address for correspondence:

César Navarro

NIBEC, Ulster University, Newtownabbey, BT37 0QB, UK

c.navarro@ulster.ac.uk 